\title{
Universal newborn hearing screening in public healthcare in South Africa: Challenges to implementation
}

\author{
J K Bezuidenhout, ${ }^{1}$ MB ChB, DCH, FCPaed, Cert Neurodevelopment, MSc; K Khoza-Shangase, ${ }^{2} \mathrm{PhD}$; \\ T de Maayer, ${ }^{1}$ MB BCh, FCPaed, MMed (Paed), Cert Gastronterol Paed; R Strehlau, ${ }^{1,3}$ MB BCh, Dip HIV, DCH, MSc \\ ${ }^{1}$ Department of Paediatrics and Child Health, School of Clinical Medicine, Faculty of Health Sciences, University of the Witwatersrand, \\ Johannesburg, South Africa \\ ${ }^{2}$ Department of Speech Pathology and Audiology, School of Human and Community Development, University of the Witwatersrand, \\ Johannesburg, South Africa \\ ${ }^{3}$ Empilweni Services and Research Unit, Rahima Moosa Mother and Child Hospital, Johannesburg, South Africa
}

Corresponding author: J K Bezuidenhout (jacqui.bez@mweb.co.za)

\begin{abstract}
Background. The implementation of early hearing detection and intervention (EHDI) remains a challenge in developing countries, despite the known benefits.

Objectives. To investigate challenges encountered during implementation of universal newborn hearing screening (UNHS) at a secondarylevel public hospital in Johannesburg, South Africa.

Methods. A prospective cohort study design that assessed the feasibility of conducting UNHS was adopted. This feasibility assessment was conducted during a 3-month period, and all challenges encountered were identified and documented. Screening time was also recorded for each neonate. Data were entered into Excel, and later analysed using Stata version 11.

Results. Of 2740 neonates born during the study period, 490 (17.9\%) were identified for screening, and distortion product otoacoustic emissions screening was conducted on $121(4.4 \%)$. The majority $(74.4 \%)$ were screened in the first 24 hours of life. Repeat screening was required in $57(47.1 \%)$ neonates, but only 20 returned for follow-up. The most important challenges to the feasibility of UNHS implementation were the insufficient number of audiologists available to provide screening, the high rate of false positive test results and the unacceptably high rates of loss to follow-up. Two modifiable factors, namely the presence of vernix caseosa in the external ear canal and high ambient noise levels, were found to have significantly influenced the screening process.

Conclusion. The identified challenges are important considerations for any successful implementation of universal screening protocols. Careful planning to mitigate the challenges will have a positive impact on EHDI initiatives in these contexts.
\end{abstract}

S Afr J Child Health 2018;12(4):159-163. DOI:10.7196/SAJCH.2018.v12i4.1522

The presence of a hearing impairment in a newborn baby may result in devastating long-term consequences. These include communication delays, emotional disturbances, cognitive deficits and, subsequently, future employment difficulties and career limitations. ${ }^{[1]}$ Failure to make the diagnosis within the first 6 months of life results in the failure to achieve vital stages in speech and language development, and consequently indicates a poorer prognosis for the individual with regard to their cognitive abilities. ${ }^{[2]}$ An expected language delay of $2-4$ years is associated with hearing loss that is detected in infants after the age of 6 months. ${ }^{[3]}$ It is therefore imperative to detect hearing impairments as early as possible in neonates and infants.

The incidence of hearing impairments in resource-poor countries is estimated at 6 per 1000 live births, compared with an estimated incidence of 2 - 4 per 1000 in industrialised countries. ${ }^{[1]}$ Prior to 1993, in the USA, only high-risk infants were assessed for hearing loss, and the auditory brainstem response (ABR) was the preferred method used. ${ }^{[4]}$ Since the recommendation from the National Institutes of Health in the USA that all infants should be screened within the first 3 months of life, all 51 states in the USA have successfully implemented universal newborn hearing screening (UNHS) programmes. ${ }^{[5]}$

Currently no formalised and standardised system of newborn hearing screening (NHS) exists at state-run hospitals in Johannesburg, South Africa (SA). Only risk-based hearing screening occurs, and this is also not systematic. Evidence shows that targeted screening misses approximately $50 \%$ of infants with hearing impairments. ${ }^{[4]}$ Therefore increased efforts are warranted towards ensuring that every baby is screened, not only those with identified risk factors.

SA has made advances towards achieving UNHS by issuing a hearing screening position statement, which is based on the 2000 position statement of the Joint Committee on Infant Hearing (JCIH) in the USA. ${ }^{[6]}$ These guidelines by the Professional Board of Speech, Language and Hearing Professions of the Health Professions Council of SA (HPCSA) $)^{[7]}$ propose that:

- all infants should be screened by no later than 1 month of age

- a full audiological examination should be offered by 3 months of age for those infants not passing the initial screening test

- infants who are confirmed as having hearing loss should receive appropriate intervention by no later than 6 months of age.

This HPCSA position statement endorses UNHS as the ideal approach to achieve early hearing detection and intervention (EHDI) goals. Early identification of hearing impairments results in the appropriate intervention being implemented before 6 months of life. ${ }^{[8]}$ Early intervention leads to improved language, cognitive and emotional development in the hearing-impaired child. A 'disabling hearing impairment' in children $<15$ years is defined by the World Health Organization as a permanent unaided hearing threshold of 31 $\mathrm{dB}$ hearing level or more in the better ear. ${ }^{[0]}$

Currently, there is limited information regarding the status of NHS programmes in SA. ${ }^{[10]}$ In 2008, 1 year after the recommendations 
from the HPCSA regarding EHDI were published, Theunissen and Swanepoel ${ }^{[5]}$ reported that only $27 \%$ of public sector hospitals in SA were implementing any form of NHS. A recent national survey of audiological services in the private healthcare sector in SA showed that there is significant delay in the overall diagnosis and provision of intervention for hearing loss. ${ }^{[1]}$ In addition to the fact that UNHS is not mandated by the SA Department of Health, there is a dearth of contextually relevant evidence regarding challenges encountered while implementing NHS in public healthcare. The present study therefore aimed to explore these challenges at a secondary level hospital in Johannesburg.

\section{Methods}

The study adhered to the Singapore Statement on Research Integrity guidelines. ${ }^{[12]}$ Ethical clearance was secured from the University of the Witwatersrand's Committee for Research in Human Subjects (ref no. M111119).

The design adopted was a prospective feasibility assessment. This was conducted at the Rahima Moosa Mother and Child Hospital (RMMCH), an academic secondary level hospital in Johannesburg. In order to provide a realistic assessment of the feasibility of implementing UNHS at this institute, only the existing staff and equipment were utilised. This context is typical of the majority of secondary level hospitals in the country; therefore the findings could be easily generalised.

\section{Study population and sample}

The study population was drawn from all neonates born at $\mathrm{RMMCH}$, using stratified systematic sampling. Selected neonates from the postnatal wards, the neonatal unit and neonatal intensive care unit (NICU) were screened. The delivery rate at RMMCH at the time of the study was 20 - 30 neonates per day. A representative sample was required, and a total of 10 neonates was considered to be a realistic number to be screened by 1 audiologist daily. To avoid enrolling a biased sample, every third neonate appearing on the birth registries in the labour ward and in the theatre was added to the list of potential neonates to be screened, if they met the eligibility criteria. The sample was stratified to be representative of the hospital's current caesarean section rate of $30 \%$, by selecting $30 \%$ of the neonates to be tested from the theatre birth register, and the remaining $70 \%$ from the labour ward registry. A total of 10 neonates was identified every day. This stratified systematic sampling was done at the start of each weekday by the researcher. It is acknowledged that this method of sampling, while it was aimed at achieving a representative sample, compromised the true nature of UNHS as defined; however, careful records of screened and non-screened neonates were kept to allow appropriate analysis of the data.

Inclusion criteria:

- all neonates born at RMMCH between 1 January 2012 and 31 March 2012

- at the time of screening, $<30$ days old.

\section{Exclusion criteria:}

- neonates not born at RMMCH (transferred in from other facilities)

- any neonate whose parent/caregiver refused to provide informed consent

- neonates who had spent more than a month in NICU, as they would have exceeded the age cut-off of 1 month.

\section{Study procedure}

Hearing screening took place during weekday working hours, and was conducted by the hospital's audiologists during the defined 3-month period. This period was dictated by the length of time allowed for the study, as this formed part of partial fulfillment requirements for a degree. The fact that hearing screening was already being conducted at the hospital as part of the audiology clinic caseload, albeit not universal, but part targeted screening, allayed the researchers' fears of possible 'teething' problems that would have been a confounding variable in this study. The screening team consisted of four audiologists. One audiologist was assigned to carry out the screening tests each week, on a rotational basis, with the screening of the neonates to be factored into their already busy schedules, bearing in mind that this was a feasibility study of UNHS implementation.

\section{Measurements}

Each neonate who was screened first underwent an otoscopic examination, to assess patency of the ear canal. Thereafter, distortion product otoacoustic emissions (DPOAE) screening, using a Natus Bio-logic AuDX with DPOAE, was conducted, giving either a 'pass' or 'refer' result. The most comprehensive protocol on the machine, with a $4 / 6$ frequency pass criterion, was used.

The audiologist conducting the screening recorded the results, indicating the time taken to screen the neonate, and record the challenges encountered, and what the plan of action would be. Unless follow-up was clinically indicated, babies receiving a 'pass' result were discharged from audiology. Newborns receiving a 'refer' result were rescreened within a month of initial screening. The rescreening procedures included an otoscopic examination, a tympanogram and a repeat DPOAE. If the results of the rescreening procedures were still inconclusive, the infant was referred for diagnostic ABR measures.

\section{Data analysis}

Data were analysed using descriptive statistics. Frequency calculations of the emergent themes were calculated, and results described qualitatively.

\section{Results \\ Study population}

All infants were $<30$ days old during data collection. Table 1 depicts the demographic profile of the sample.

Of the neonates included in the study, 79 (65.3\%) were born via normal vaginal delivery, with $2(1.7 \%)$ requiring assisted delivery and 40 (33\%) born via caesarean section. This delivery distribution pattern was consistent with the hospital's anticipated caesarean section rate of $30 \%$. During the screening period, there were 855 caesarean section deliveries, making up $31.2 \%$ of the total deliveries. Of the 121 study participants screened, $90(74.4 \%)$ were HIV-unexposed, 29 (24\%) HIV-exposed, and 2 (1.6\%) born to mothers who had, at the time of screening, not yet been tested for HIV.

During the study period, 2740 neonates were born at RMMCH. A total of 490 neonates were identified and assigned study numbers. Of the sample group, two mothers refused consent for their infants to be screened. A total of 121 neonates were screened, representing $24 \%$ of the identified neonates. A total of 369 neonates $(75.3 \%)$ were identified for potential screening, but did not undergo screening. This ommission was primarily due to the lack of staff to perform the screening, and is further discussed under the challenges experienced. Therefore, of the 2 740 neonates born at $\mathrm{RMMCH}$ during the study period, $4.4 \%$ underwent screening.

\section{Screening results}

As depicted in Fig. 1 below, 57 of the 121 participants (47.1\%) had a 'refer' result at the first screening, while the remainder were discharged, as they passed the screening bilaterally. Of the 57 due to 
come back for repeat DPOAE, only 20 (35.1\%) returned for followup. Upon repeat DPOAE, 18 of the $20(90 \%)$ passed, and therefore were subsequently discharged, and $2(10 \%)$ required $A B R$, as per the research protocol. Ultimately only 83 neonates were successfully screened, as 38 individuals were lost to follow-up.

\section{Challenges identified during screening}

Table 1. Demographic and medical profile of the sample $(N=121)$

\begin{tabular}{ll}
\hline Factor & Neonates screened, $\boldsymbol{n}$ (\%) \\
\hline Birth weight, g & $16(13.2)$ \\
$<2500$ & $105(86.8)$ \\
$\geq 2500$ & $79(65.3)$ \\
Type of delivery & $40(33)$ \\
NVD & $2(1.7)$ \\
Caesarean section & \\
Assisted delivery & $29(24)$ \\
HIV exposure & $90(74.4)$ \\
Yes & $2(1.6)$ \\
No & \\
Unknown & $2(1.7)$ \\
ICU admission after birth & $119(98.3)$ \\
Yes & \\
No & $6(5)$ \\
Apgar scores & $115(95)$ \\
1 minute Apgar $\leq 5$ & 0 \\
1 minute Apgar $>5$ & $121(100)$ \\
5 minute Apgar $\leq 5$ & 0 \\
5 minute Apgar $>5$ & $121(100)$ \\
10 minute Apgar $\leq 5$ & \\
10 minute Apgar $>5$ & \\
NVD = normal vaginal delivery; ICU $=$ intensive care unit. & \\
&
\end{tabular}

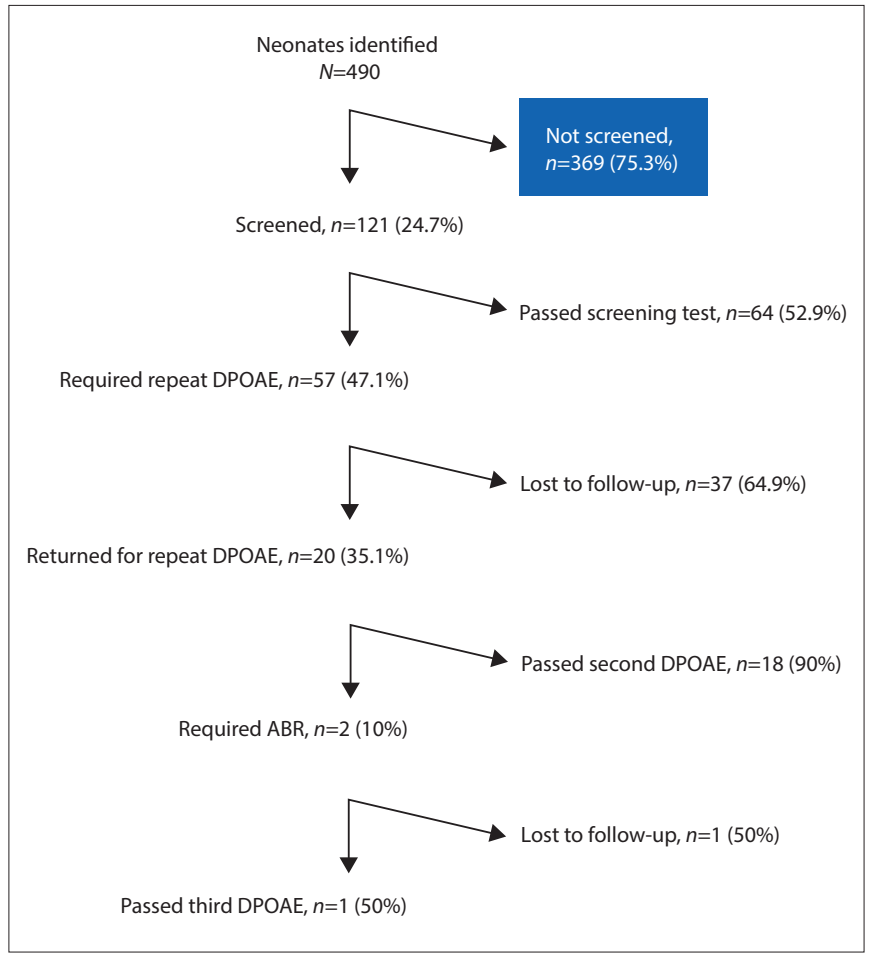

Fig. 1. Neonate hearing screening outcomes. (DPOAE = distortion product otoacoustic emissions screening; $A B R=$ auditory brainstem response.)
Challenges identified during screening included factors related to the actual screening process itself, as well as other logistical factors. The mean time taken to screen each neonate was 11 minutes 17 seconds (range 5 - 40 minutes), and the median time taken to screen was 10 minutes. Thirty-four neonates had a screening time of longer than 11 minutes, and 11 (32\%) of these neonates had no identifiable challenges contributing towards their prolonged screening time.

Challenges (Table 2) included equipment failure, noise interference, and vernix caseosa in the external auditory canal.

- Equipment failure was identified as a challenge in $5(4.1 \%)$ cases, 2 of which required repeat DPOAEs.

- Noise interference was identified in 39 (32.2\%) cases, and $27(69.2 \%)$ of these required a repeat DPOAE (odds ratio (OR) 2.25, 95\% CI $1.14-4.44 ; p=0.001)$. Half of these neonates $(14 / 27,52 \%)$ also had a finding of vernix caseosa in their ears at the initial screen.

- Vernix Caseosa was identified as a challenge complicating screening. Its presence was documented in 86 (71\%) neonates, but was a challenge complicating screening in only $40(33 \%)$ neonates, with $39(97.5 \%)$ requiring a repeat DPOAE (OR 0.015, $95 \%$ CI $0.002-0.1155, p<0.0001)$.

- Other challenges included crying $(n=4,3.3 \%)$, the screening session being interrupted $(n=1,0.8 \%)$ and a baby who was hiccupping during the session $(n=1,0.8 \%)$.

\section{Other challenges identified during the study}

During the screening period, 121 neonates out of a total of 2740 live birth deliveries (4.4\%) were screened. Challenges encountered included:

- High patient-assessor ratio/limited coverage - a team of 4 audiologists was available to conduct the screening. However, 1 audiologist resigned during the study period. Prior to the decrease in the staff complement, the audiologist on duty would see, on average, 12 neonates per week. This decreased in the third month of screening, to 7 neonates per week (Fig. 2).

- Technical difficulties were encountered with the DPOAE machine in the second week of screening, requiring repair, which took 3 weeks. Screening could only recommence once an OAE machine had been borrowed from the university's audiology department, in the fourth week of screening. Another technical difficulty was that at the time of the screening, the ABR machine at the referral hospital was also being repaired. This had a negative impact on the 2 neonates requiring $\mathrm{ABR}$ testing after not passing DPOAE screening.

- The early discharge of neonates born via normal vaginal delivery also created a challenge. Mothers and infants are typically discharged home within 6 - 8 hours after delivery, if no complications exist.

\section{Discussion}

This study highlighted many of the challenges present in this context that are preventing the implementation of UNHS.

The implementation of a UNHS programme at a secondary institute was found to be unattainable in the study's setting with the available staff and equipment.

Table 2. DPOAE screening results $(N=121)$

\begin{tabular}{ll}
\hline Initial test result & $\boldsymbol{n}, \boldsymbol{\%}$ \\
\hline Pass & $64(53 \%)$ \\
Refer & $57(47 \%)$ \\
Difficulties encountered during screening & \\
$\quad$ Vernix & $40(33 \%)$ \\
Noise interference & $39(32 \%)$ \\
Equipment & $5(4.1 \%)$ \\
DPOAE = distortion product otoacoustic emissions. &
\end{tabular}




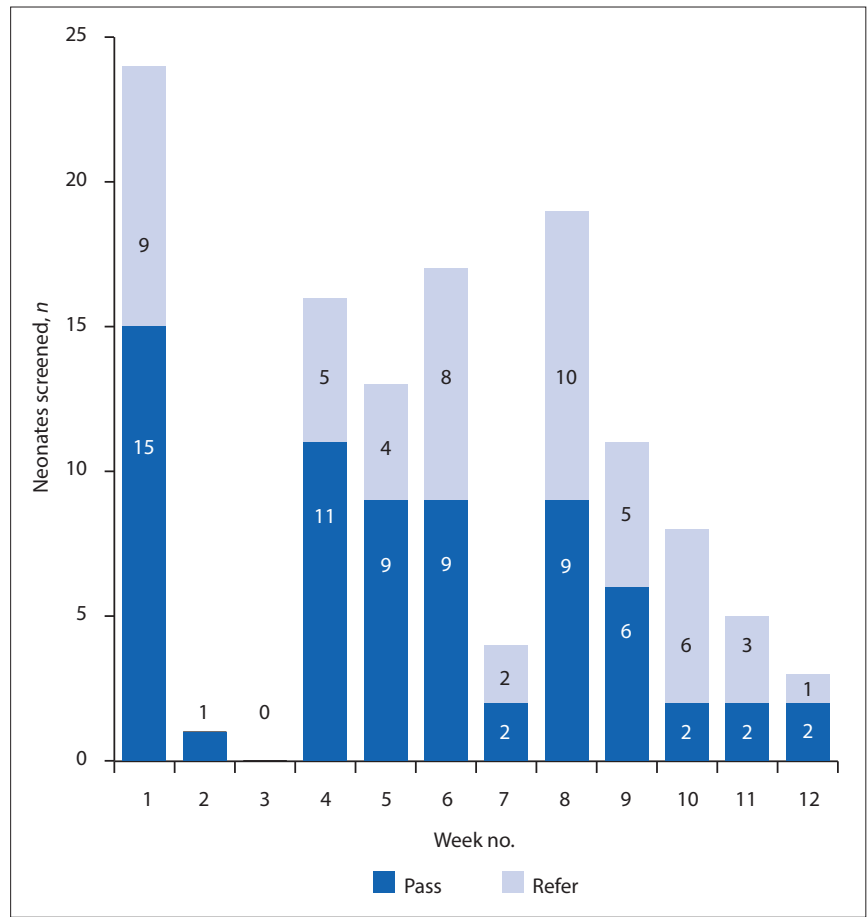

Fig. 2. Neonates screened per week $(\mathrm{N}=490)$; passed test $v$. referred for repeat testing.

The primary challenge was the limited number of audiologists available to perform the screening. With 2740 neonates being delivered during the screening period, 228 neonates would need to be screened per week, and 45 neonates would require screening daily during a 5-day working week. Audiologists employed in the state sector do not currently receive financial remuneration for any overtime work, and so screening had to occur during regular working hours. An alternative to audiologists being solely responsible for the hearing screening would be to involve nursing staff or trained screeners. This would alleviate the problem of audiologists being required to conduct screening after hours, as most nursing staff work in shifts and so would be available for after-hours screening. This would also ensure that the aim of UNHS, to screen all newborns just after birth or before discharge from hospital, is achieved. ${ }^{[8]}$ In Lagos, Nigeria, a 2-week training period for non-specialist staff in the use of transient evoked otoacoustic emissions screening was deemed feasible. The study reported that $98.7 \%$ of all eligible newborns were screened before being discharged from the hospital. ${ }^{[13]}$ A similar study in SA, where clinic nurses were trained to screen infants with DPOAEs, was successful in achieving a high follow-up rate for those requiring repeat screening, although it only managed to screen $32.4 \%$ of infants eligible for screening. ${ }^{[14]}$

The next challenge identified in this study was one of screening equipment. In the second week of screening, the OAE machine malfunctioned, and was sent to the manufacturer for repair; this took 3 weeks. This technical complication was unexpected, but highlights the fact that in order for a unit to have a successful UNHS programme, there should be more than one piece of equipment available. This recommendation applies to diagnostic ABR equipment as well, as this forms part of the important measures required for EHDI to be successfully implemented..$^{[6,7]}$

Although these physiological forms of testing are the approved screening methods, alternative methods may be utilised in resourcepoor settings. ${ }^{[715]}$ Behavioural observation testing has been shown to have a high sensitivity and specificity, as well as being quick and inexpensive to perform, although its specificity and sensitivity has been found lacking when compared with objective electrophysiologic measures. ${ }^{[16]}$ Historically, this form of testing was associated with infants aged 6 - 9 months, which may be too late, considering that the $2007 \mathrm{JCIH}$ guidelines on detecting hearing impairments recommend that hearing loss be identified by 3 months of age. ${ }^{[17]}$ Behavioural observation testing could be of limited use in the interim for developing countries.

The next challenge identified was the influence of ambient noise, which resulted in a statistically significant number of children requiring repeat DPOAEs screening. The ideal hospital ward should be a setting of rest for a patient, especially in a postnatal ward where mothers and babies are rooming-in together. The reality is that the ambient noise levels recorded in the wards of a developing country's hospital were found to typically range between 61.0 and $90.5 \mathrm{~dB} .^{[18]}$ According to the World Health Organization, an ideal ambient noise level in a hospital ward is $30 \mathrm{~dB} \cdot{ }^{[19]} \mathrm{A}$ dedicated screening area with ambient noise levels not exceeding $50-55 \mathrm{~dB}$ is required so as to minimise the number of false positive test results. ${ }^{[20]}$ False positive 'refer' results may cause the caregivers undue stress, and result in their having to return to the hospital for a repeat screening test.

The next challenge influencing successful implementation of UNHS in the current study was vernix caseosa, a physiological substance produced by the fetus in utero. ${ }^{[21]}$ Owing to the sticky nature of vernix, it can be quite difficult to remove from a neonate's ear. Otoscopic examination revealed that $86(71 \%)$ neonates had vernix in their ears, but in only $40(46 \%)$ was this identified as a challenge by the screener. Forty-five percent of neonates with vernix caseosa required repeat DPOAE. The removal of vernix caseosa and other debris from the ear canal of neonates has been shown to improve pass rates when conducting OAEs. ${ }^{[22,23]}$

Khoza-Shangase et al. ${ }^{[24]}$ recommend that in order for more neonates to be assessed, and for confounding factors such as the presence of vernix caseosa to be minimised, screening should take place on day 3 of life, at the midwife outpatient unit. This would be difficult to implement in the current study's facility, as neonates who are born vaginally there are typically discharged within a couple of hours of delivery. Similar findings were reported by Tsui et al., ${ }^{[25]}$ who described delaying diagnostic ABR by up to 50 days post-delivery. The disadvantages of delaying the hearing screening are that there could potentially be a delay in the detection of hearing loss, as well as potential emotional stress to the caregivers as they await the hearing assessment. ${ }^{[25]}$ This possible anxiety from delaying the screening needs to be weighed against the potential anxiety caused by a false positive finding, which may inhibit bonding between mother and child. ${ }^{[26]}$

The last challenge identified in this study was early discharge of neonates from the ward. Screening neonates born after hours via normal vaginal delivery was an anticipated difficulty, as these mothers are typically observed for $6-8$ hours post-delivery, and then discharged. A possible solution to this would be the audiologists having a larger team, who could then screen at two time intervals during the day, or alternate healthcare professionals being trained to conduct hearing screening. ${ }^{[27,28]}$ The second group of neonates who were not screened was those who were born over the weekends and discharged home before being enrolled into the study. This limitation was a major factor when assessing the feasibility of UNHS.

The aforementioned challenges require careful attention during the planning and implementation of UNHS in developing-country contexts, where resource constraints dictate priorities.

\section{Conclusion}

Current findings revealed that conducting a UNHS programme was not feasible at a secondary level hospital in Gauteng, SA. These findings raise important implications around planning, human resource allocation, budgeting for equipment, as well as training of non-audiologists for successful implementation of 
UNHS programmes within this context. Current findings should be interpreted within the identified study's methodological limitations. The main limitation of this study was that the time period used for data collection was limited to three months. It is believed that a longer time frame might have influenced the current findings. The second limitation is that the study was in one hospital where resources are significantly better than in a number of other hospitals in the country; therefore, generalisability of the findings is limited. These limitations, therefore, raise implications for future studies.

Acknowledgements. The authors would like to acknowledge the audiologists at RMMCH for their role in the hearing screening of neonates in this study.

Author contributions. JKB and KK-S conceptualised the study and wrote the first draft, with JKB conducting data collection, under the supervision of KK-S and TdM. TdM and RS were involved in co-writing the manuscript.

Funding. None.

Conflicts of interest. None.

1. Olusanya BO. Priorities for early hearing detection and intervention in sub-Saharan Africa. Int J Audiol 2008;47(S1):S3-S13. https://doi. org $/ 10.1080 \% 2$ F 14992020802287143

2. Swanepoel D, Delport S, Swart J. Universal newborn hearing screening in South Africa - a first-world dream? S Afr Med J 2004;94(8):634-635.

3. Yoshinaga-Itano C, Sedey AL, Coulter DK, Mehl AL. Language of early- and later-identified children with hearing loss. Pediatrics 1998;102(5):1161-1171. https://doi.org/10.1542\%2Fpeds.102.5.1161

4. Kanne TJ, Schaefer L, Perkins JA. Potential pitfalls of initiating a newborn hearing screening programme. Arch Otolaryngol Head Neck Surg 1999;125(1):28-32. https://doi.org/10.1001\%2Farchotol.125.1.28

5. Theunissen M, Swanepoel DW. Early hearing detection and intervention services in the public health sector in South Africa. Int J Audiol 2008;47(S1):S23-S29. https://doi.org/10.1080\%2F14992020802294032

6. Joint Committee on Infant Hearing. Year 2000 position statement: Principles and guidelines for early hearing detection and intervention programs. Am J Audiol 2000;9(1):9-29. https://doi.org/10.1044\%2F1059-0889\%282000\%2F005\%29

7. Health Professions Council of South Africa (HPCSA). Professional Board for Speech, Language and Hearing Profession: Early Hearing Detection and Intervention Programmes in South Africa. Position Statement Year 2007. Pretoria: HPCSA,2007:1-42. https://doi.org/10.1044\%2Fpolicy.ps2007-00281

8. Joint Committee on Infant Hearing. Year 2007 position statement: Principles and guidelines for early hearing detection and intervention programmes. Pediatrics 2007;120(4):898-921. https://doi.org/10.1542\%2Fpeds.2007-2333

9. World Health Organization. Primary Ear and Hearing Care Training Resource. Advanced Level. Geneva: WHO, 2006. http://www.who.int/pbd/deafness/ activities/hearing_care/en/ (accessed 22 November 2018).

10. Swanepoel D, Storbeck C, Friedland P. Early hearing detection and intervention in South Africa. Int J Pediatr Otorhinolaryngol 2009;73(6):783-786. https://doi. org/10.1016\%2Fj.ijporl.2009.01.007

11. Meyer ME, Swanepoel de W, Le Roux T. National survey of paediatric audiological services for diagnosis and intervention in the South African private healthcare sector. S Afr J Commun Disord 2014;61(1):e1-e8. http://doi. org/ 10.4102/sajcd.v61i1.62
12. Lucas G. Singapore statement on research integrity. Sri Lanka J Child Health 2010;39(4):126-127. https://doi.org/10.4038\%2Fsljch.v39i4.2476

13. Olusanya B. Hospital-based universal newborn hearing screening for early detection of permanent congenital hearing loss in Lagos, Nigeria. Int J Pediatr Otorhinolaryngol 2008;72(7):991-1001. https://doi.org/10.1016\%2Fj. ijporl.2008.03.004

14. Friderichs N, Swanepoel D, Hall JW. Efficacy of a community-based infant hearing screening programme utilising the existing clinic personnel in Western Cape, South Africa. Int J Pediatr Otorhinolaryngol 2012;76(4):552-559. https:// doi.org/10.1016\%2Fj.ijporl.2012.01.015

15. Olusanya BO. Highlights of the new WHO Report on Newborn and Infant Hearing Screening and implications for developing countries. Int J Pediatr Otorhinolaryngol 2011;75(6):745-748. https://doi.org/10.1016\%2Fj. ijporl.2011.01.036

16. Kuki S, Chadha S, Dhingra S, Gulati A. The role of current audiological tests in the early diagnosis of hearing impairment in infant. Ind J Otolaryngol Head Neck Surg 2012;65(3):244-250. https://doi.org/10.1007\%2Fs12070-012-0558-x

17. Kamal N. Newborn hearing screening: Opportunities and challenges. Egyp J ENT Allied Sci 2013;14(2):55-58. https://doi.org/10.1016\%2Fj. ejenta.2013.01.002

18. Olusanya B. Ambient noise levels and infant hearing screening programs in developing countries: An observational report. Int J Audiol 2010;49(8):535541. https://doi.org/10.3109\%2F14992021003717768

19. Berglund B, Lindvall T, Schwela DH, Team WHOOaEH. World Health Organization 1999 Guidelines for Community Noise. Geneva: World Health Organization, 1999 (updated 8 November 2014). http://www. who.int/iris/handle/10665/66217\#sthash.83492aam.dpuf (accessed 22 November 2018).

20. Rhoades K, McPherson B, Smyth V, Kei J, Baglioni A. Effects of background noise on click-evoked otoacoustic emissions. Ear Hearing 1998;19(6):450-462. https://doi.org/10.1097\%2F00003446-199812000-00006

21. Singh G, Archana G. Unraveling the mystery of vernix caseosa. Ind J Dermatol 2008;53(2):54-60. https://doi.org/10.4103\%2F0019-5154.41645

22. Chang K, Vohr B, Norton S, Lekas M. External and middle ear status related to evoked otoacoustic emission in neonates. Arch Otolaryngol Head Neck Surg 1993;119(3):276-282. https://doi.org/10.1001\%2Farchot ol.1993.01880150024004

23. Doyle KJ, Rodgers P, Fujikawa S, Newman E. External and middle ear effects on infant hearing screening test results. Otolaryngol Head Neck Surg 2000;122(4):477-481. https://doi.org/10.1016\%2 Fs0194-5998\%2800\%2970087-8

24. Khoza-Shangase K, Harbinson S. Evaluation of universal newborn hearing screening in S Afr Prim Care 2015;7(1):1-12. https://doi.org/10.4102\%2Fphcfm. v7il.769

25. Tsui PW, McPherson B, Wong EC, Ng IH. Infant hearing screening: Effects of timeline. Clinical Otolaryngol 2008;33(2):108-112. https://doi. org/10.1111\%2Fj.1749-4486.2008.01663.x

26. Olusanya BO, Luxon L, Wirz S. Benefits and challenges of newborn hearing screening for developing countries. Int J Pediatr Otorhinolaryngol 2004;68(3):287-305. https://doi.org/10.1016\%2Fj.ijporl.2003.10.015

27. Kanji A, Khoza-Shangase K. Feasibility of newborn hearing screening in a public hospital setting in South Africa: A pilot study. S Afr J Communication Disord 2016;63(1):a142. https://doi.org/10.4102/sajcd.v63il.150

28. Khoza-Shangase K, Kanji A, Petrocchi-Bartal L, Farr K. Infant hearing screening from a developing country context: Status from two South African provinces. S Afr J Child Health 2017;11(4):159-163. https://doi.org/10.7196/ SAJCH.2017.v11i4.1267

Accepted 25 April 2018. 